\title{
Abstraction Learning
}

\author{
Joxan Jaffar, Jorge Navas, and Andrew Santosa \\ National University of Singapore
}

\begin{abstract}
A state-of-the-art approach for proving safe programs is CounterExample-Guided Abstraction Refinement (CEGAR) which performs a "abstraction-verificationrefinement" cycle by starting with a coarse abstract model that is then refined repeatedly whenever a counterexample is encountered. In this work, we present a dual approach which starts with a concrete model of the program but progressively abstracts away details but only when these are known to be irrelevant. We call this concept Abstraction Learning (AL). In order to deal with loops, our algorithm is encapsulated in an iterative deepening search where, because of a particular depth bound, abstraction is forced upon loops. This abstraction will correspond to the strongest loop invariant we can discover. As in CEGAR, this abstraction is of a speculative nature: if the proof is unsuccessful, the abstraction is removed and we initiate a new attempt using a new depth bound.

A key difference between AL and CEGAR is that AL detects more infeasible paths in the state-space traversal phase. We argue that this key feature poses unique benefits, and demonstrate the performance of our prototype implementation against the state-of-the-art BLAST system.
\end{abstract}

